Session TC4-1

\title{
Design Calculator for Quick Estimation of Concrete and Reinforcement in the Flat Plate Slab
}

\author{
Mr. Vardhaman V. Bora \\ Dr. Mohammed E. Haque \\ Department of Construction Science \\ Texas A\&M University, College Station, TX 77843-3137
}

\begin{abstract}
Design analysis and cost estimation play important roles in preconstruction activities. Accurate estimates are necessary to keep decisions within budget and to help builders determine which of their designs has the best chance of "getting the project." This means builders must have at their disposal an accurate means to determine which of their designs is the most effective in terms of cost, performance, and reliability. However, standard approaches to measure out these parameters are often too time-consuming or too expensive to allow comprehensive comparisons of alternatives. This paper looks at how computer aided models coupled with a design knowledge database can accelerate the design and estimate process. It further discusses the procedure for developing such a knowledge database, which inputs simple geometric inputs and outputs detailed reinforcement and concrete requirements. The model is designed for the flat plate slab, as flat slab is being widely used especially when requirements are of minimum floor to floor height or clear long spans (MacGregor, James Wight, 2005). Microsoft Excel is used to house the database as per the design method and respective ACI code. Using this model, a person from non-engineering background can also provide accurate preliminary design with detail layout and realistic quantity estimation of reinforcement and concrete.
\end{abstract}

\section{Introduction}

The major concern during preconstruction is accurate preliminary design, preliminary quantity take-off or cost estimate and comparison of design alternatives in limited time and budget.

Design especially the structural analysis is governed by loading condition; geometry and material properties of its associated component. All the computation is code specific, change in one of the parameter changes the values to be taken from code and associated design as all calculations are interconnected. Final design can be obtained through various combination of material and geometrical properties for a given loading conditions. There would be multiple designs which can satisfy requirement of project (Mousa, Tabatabai- Gargari, Elzarka \& Hazem, 1999). Computation of cost associated with the design will give criteria for comparison, and choose the optimal one. Preliminary estimation will also give owner idea to determine if they meet their budget; otherwise project is redesigned for reduced cost. 
As we have seen that decision ultimately depends on estimated value it is important that it should be accurate and reliable. Accurate cost estimation is achieved through detail design and providing exact values for estimation.

Mousa, et al. (1999) found that value analysis can be used to identify alternative design approaches for satisfying the requirements of the project while lowering costs and ensuring technical competence in performance.

\section{Automation of flat slab design and estimation}

Flat plate slabs are slabs of uniform thickness without beam. Flat slab is most economical choice of floor system and now it is being widely used in apartments or multistory buildings especially when requirements are of minimum floor to floor height and clear long spans.

Advantages of Flat Slab Construction, Karve, Shah (2003):

1. It provides a large clear ceiling height

2. The construction is simple and economical because of the simplified form work, the ease of placement of reinforcement.

3. the plain ceiling gives an attractive and pleasing appearance

4. In absence of beams, provision of acoustical treatments is easy

5. In general flat slab construction is economical for spans up to $10 \mathrm{~m}$ and relatively light loads.

There are various standard design methods, such as, 'Direct Design Method', Strip Design Method' and Finite Elements Method', which are used for the flat slab design (British Cement Association, 2001). All of these methods involve large number of computation and many specifications to follow as per the ACI codes. It is very time consuming if it is done by traditional approach and also creates limitation on consideration of alternatives. There is also restriction on money that can be spent as the analysis is before getting the project.

Computer-aided applications can expedite the process of design analysis and estimation. Sophisticated software are available which are proficient in design analysis or estimation (Mousa, et al., 1999). But there are very rare tool which can encompass both the features of design and estimation along with facilitating the decision making process. There are various other drawbacks associated with the existing software. Availability in common, compatibility, and ease in use are few to list.

In any of these software applications there is no such facility for automation of flat slab. So this makes flat slab as the ideal choice for automating preconstruction activities.

\section{Use of Microsoft Excel for analysis of flat slab}

We have discussed requirement of design and estimation that design has lot of computation and code specifications while estimation has to take value from design and need to be accurate. So the platform for automation should be such that it will have programming capabilities, wide range of mathematical and logical functions, and options for drawing schematic sketches. Along 
with that it is to be user friendly and most commonly used in construction industry to overcome the limitation of the other software.

Among the several software development platforms and languages, Microsoft's excel is the one of the best powerful tool which is suitable for the analysis and above kind of requirements.

Survey conducted by the Construction Financial Management Association in 2008 found that Excel is the most popular estimating software among construction professional that have increasingly computerized their operations. Many people have started to put Microsoft Excel as they may already be familiar with it; it's inexpensive, and widely available.

\section{Developing knowledge base system}

Excel spreadsheet is developed into a knowledge base system by providing it with code specification and required design parameters. It takes simple inputs from user like material properties, load conditions and geometrical properties of slab and column. A typical platform for user in put is shown in Figure 1.

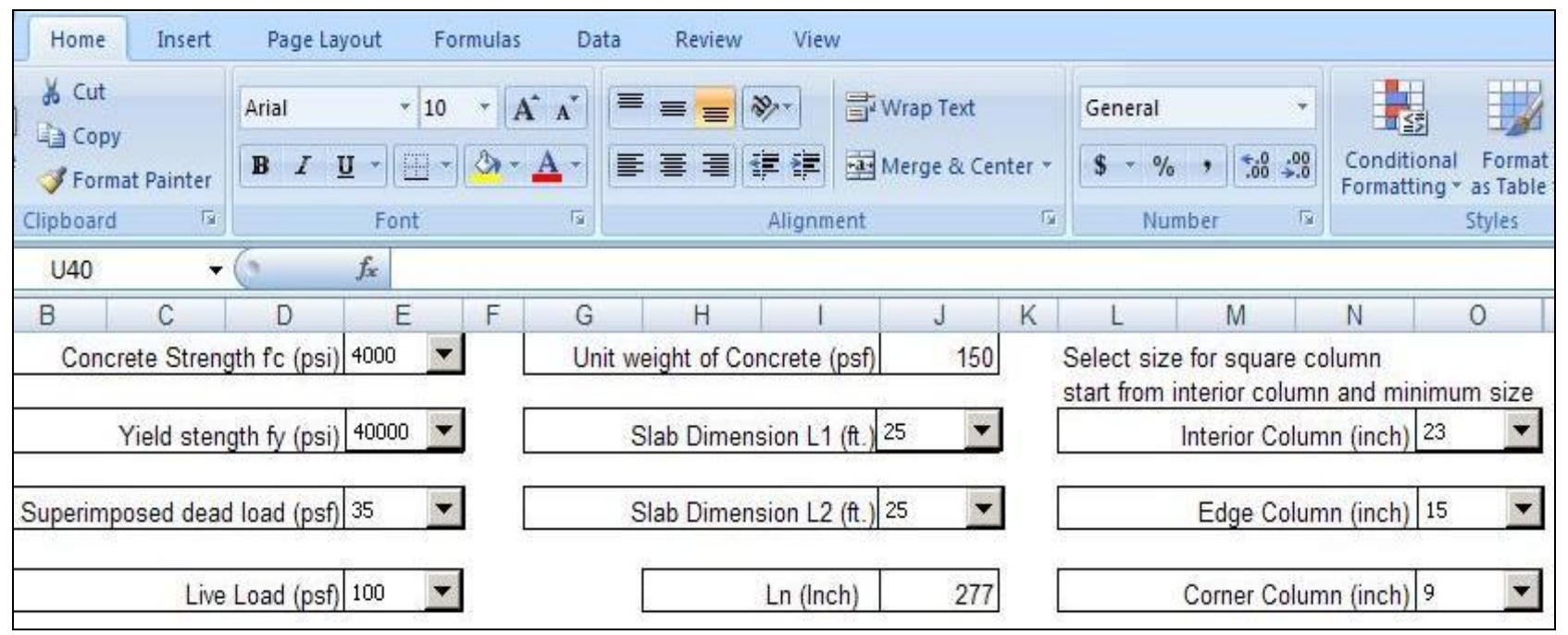

Figure 1 User Input sheet

It provides options for concrete strength of 3000, 4000, and 5000 psi; yield strengths of steel of 40000, 60000, and 75000 psi as per the expected standard of concrete and steel.

Range for live load is 10 to 100 psf, while range for superimposed load is 15 to 35 psf, depending on the purpose of building and its relative occupancy.

Slab can be square or rectangular with a maximum dimension as 26 feet and minimum of $16 \mathrm{ft}$. as flat slab is economical for span less than $26 \mathrm{ft}$ and aspect ratio less than 2 . Square columns can be chosen from cross section size as 9' $\mathrm{X9}$ '” to 26 ' $\mathrm{X} 26$ ". This range is determined out of the consideration of maximum and minimum amount of loading that will be transferred on column at any location. 
Computational steps are coded in excel as per the ACI 318 - 08, 2008 Direct Design Method. In order to that it should work as per the code, rules are expressed in terms of logical functions such as if-then conditions. It will typically give concrete volume and reinforcement along with the layout and schedule for the steel bars. Reinforcement is calculated with its detail in terms of size, no., length, weight and placing. It is a simple calculator which will easily allow consideration of multiple design alternatives to a non-structural personal.

From the basic project information such as, area in square footage and use of building, parameters such as slab dimension and loading conditions can be made fix. User can make permutation and combination for rest of parameters starting from lowest of the range value. As this system is given with knowledge of code specifications and design method, it will guide user about their selection. The basic concept behind is, all the properties will finally decide the load that is transferred from slab to column, so when user will select the column size system will check whether the column of selected size can sustain the load from slab or not. In following section you will find more details on this.

\section{Validating the selection through column size}

Design of flat slab is to be done for 3 different cases (Mehdi, Darvas, 2007),

- Interior panel

- Exterior panel with one edge unrestrained and

- Exterior panel with two edges unrestrained.

For each case slab thickness is found out depending on yield strength and maximum of them is taken as common slab thickness for all the panels. With this thickness and other selected parameters factored load per square feet is calculated. For the columns depending on the location, the area that it is supporting will vary and so as the load that it has to carry. Figure 2 describes the loading area on each column.

As we can see there are three types of column, Interior, edge and corner. Load coming on this column reduces from interior to edge and least in corner column. So the requirement of size of column also reduces and hence there is no need to provide a uniform column size throughout.

User is provided with an option to select column size for all the three locations one by one. Now from column cross section and concrete properties, the minimum value of shear force which will be always sustained by selected cross section is computed. From above we also know loading area and factored load. Simple multiplication will give actual shear coming on column. Know system will check for shear and decide if column size is enough or not.

As shown in Figure 3, end user will see only the pop pup error message. It will be 'unsatisfactory' if either of selected cross section is below the requirement. System will further guide user, to increase the column size, or it will ask to improve the strength of steel or concrete if column size could not be extended beyond certain limit.

Thus the system is trained to make sure that the design is safe at all the time, with whatever changes you make in your selection. 


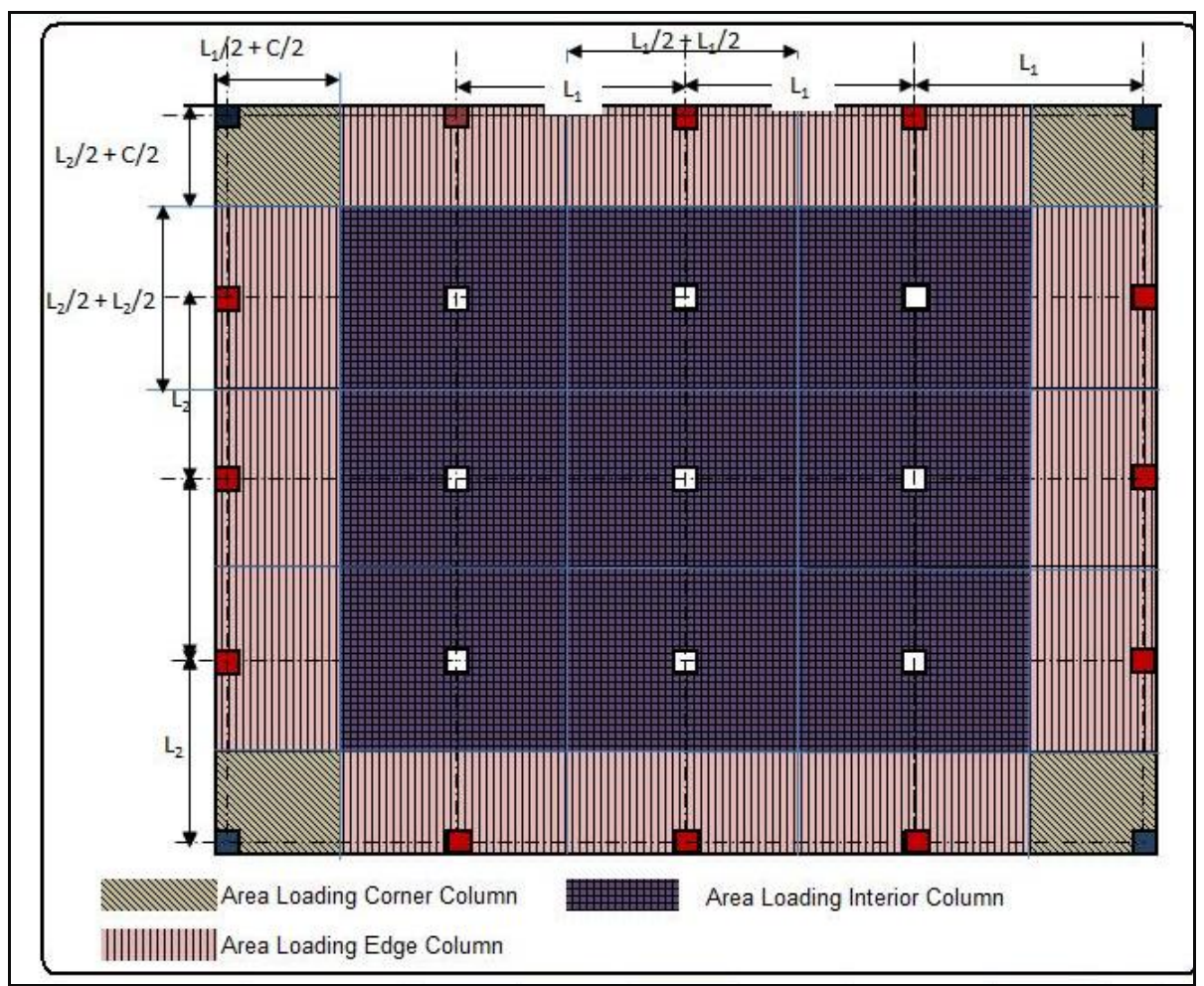

Figure 2 Distribution of slab load on column

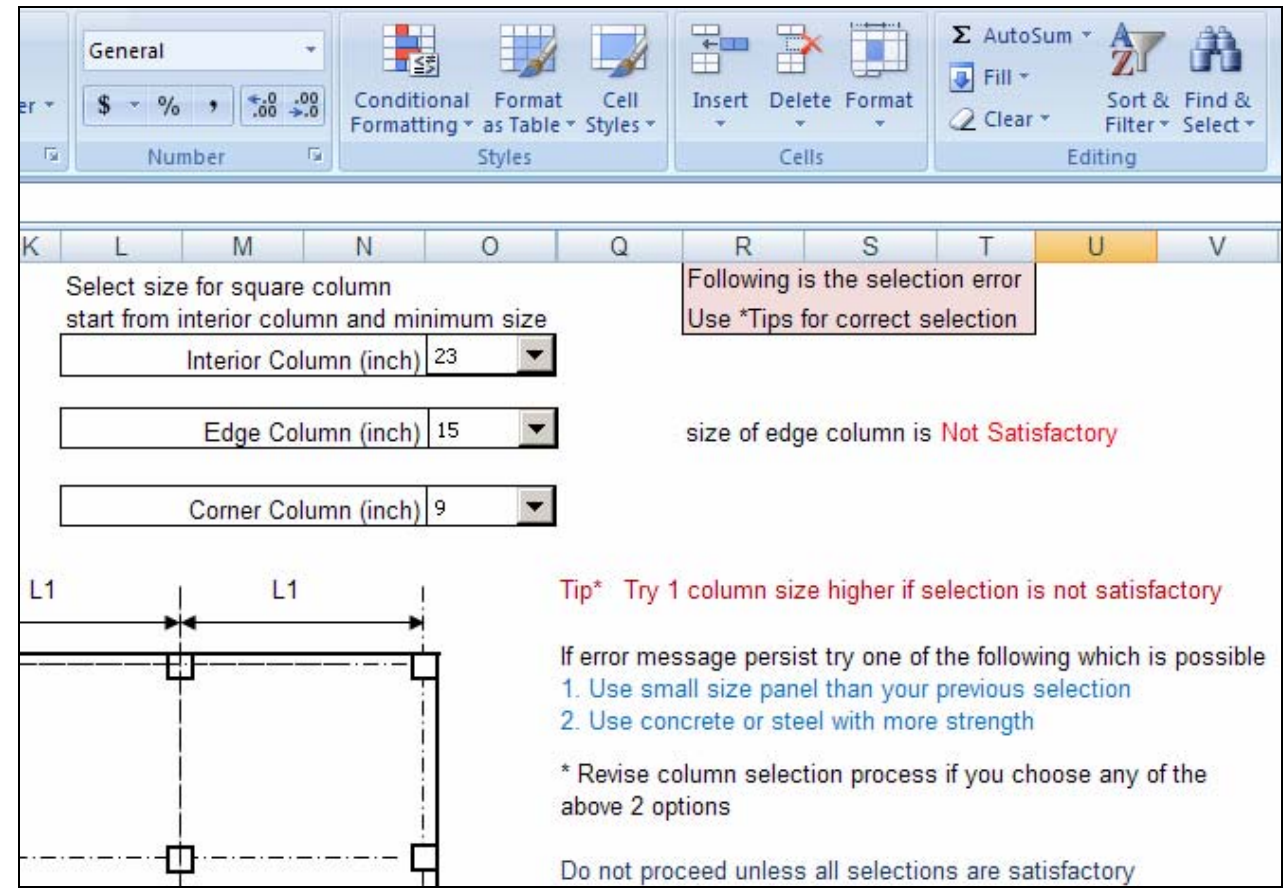

Figure 3 Typical error message if any of the column is undersized 


\section{Determining the required reinforcement}

As we have discussed in previous section that flat slab is designed for 3 cases, reinforcement is also calculated separately. System is provided with all design steps. It takes required parameter, computes all intermediate values, and finds required reinforcement. An example of reinforcement detailing and estimation is illustrated in Figure 4.

In flat slab, reinforcement is calculated for column strip and middle strip. Following all the encoded design steps, system will calculate the area of steel required in top and bottom of bar for both strip as displayed in schematic sketch of Figure 4.

User will select size of bar from the standard sizes listed in drop down box. In the next columns spacing between the bars and respective number of bars would be generated accordingly as we can see in the table of figure 4 .

Now user can have same size of bar for entire span or change the size of bar to have same spacing. System would adjust the required number of bars and spacing accordingly. It will further compute the total length and so as weight of bars for top and bottom reinforcement of column and middle strip. Total will give steel requirement of that and similar panels. Similarly steel required is computed for rest of the two cases.

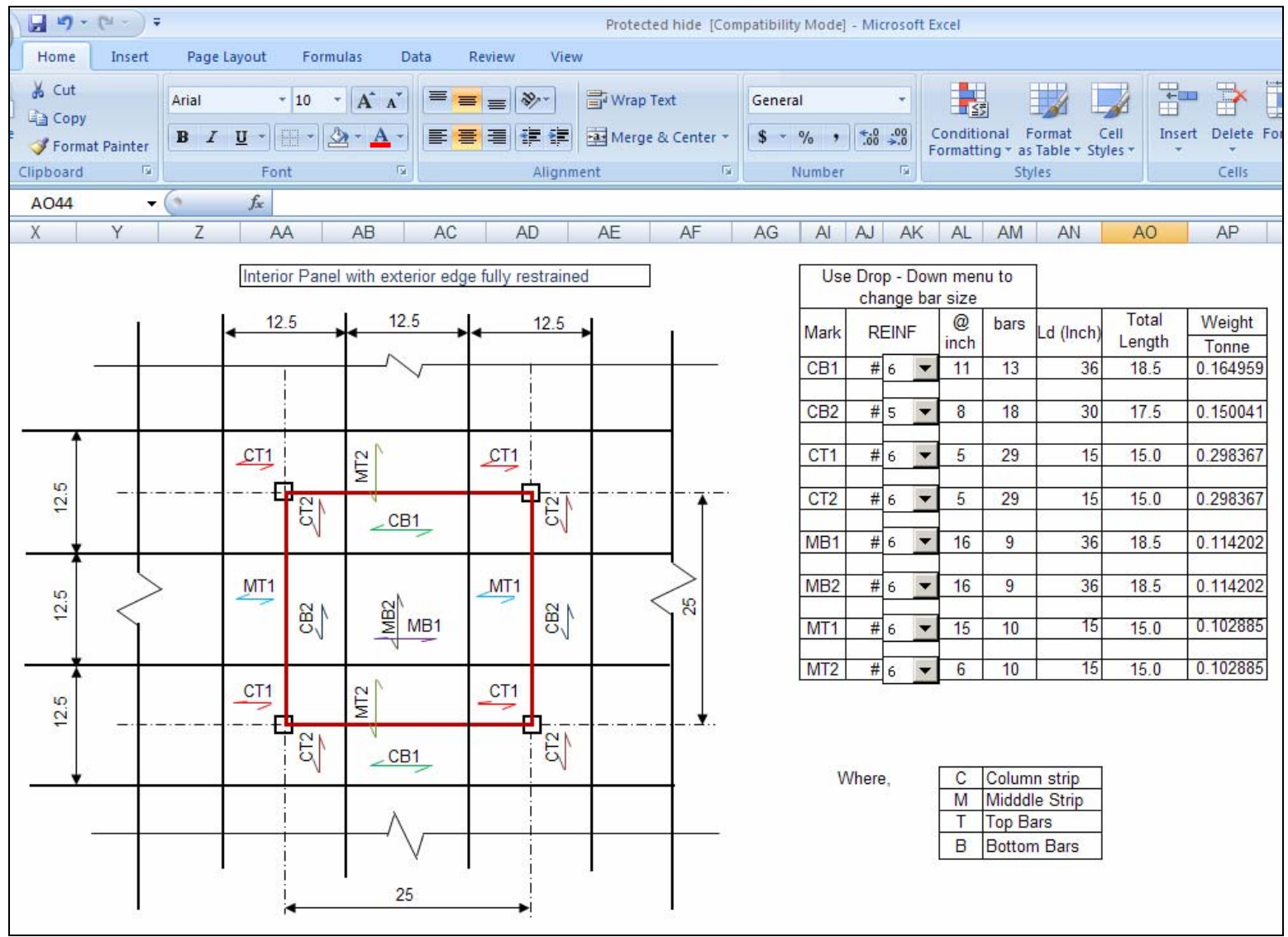

Figure 4 Reinforcement detailing for interior panel with exterior edge fully restrained 
Now user can have same size of bar for entire span or change the size of bar to have same spacing. System would adjust the required number of bars and spacing accordingly. It will further compute the total length and so as weight of bars for top and bottom reinforcement of column and middle strip. Total will give steel requirement of that and similar panels. Similarly steel required is computed for rest of the two cases.

Summary report would be displayed on input sheet as shown in figure 5.

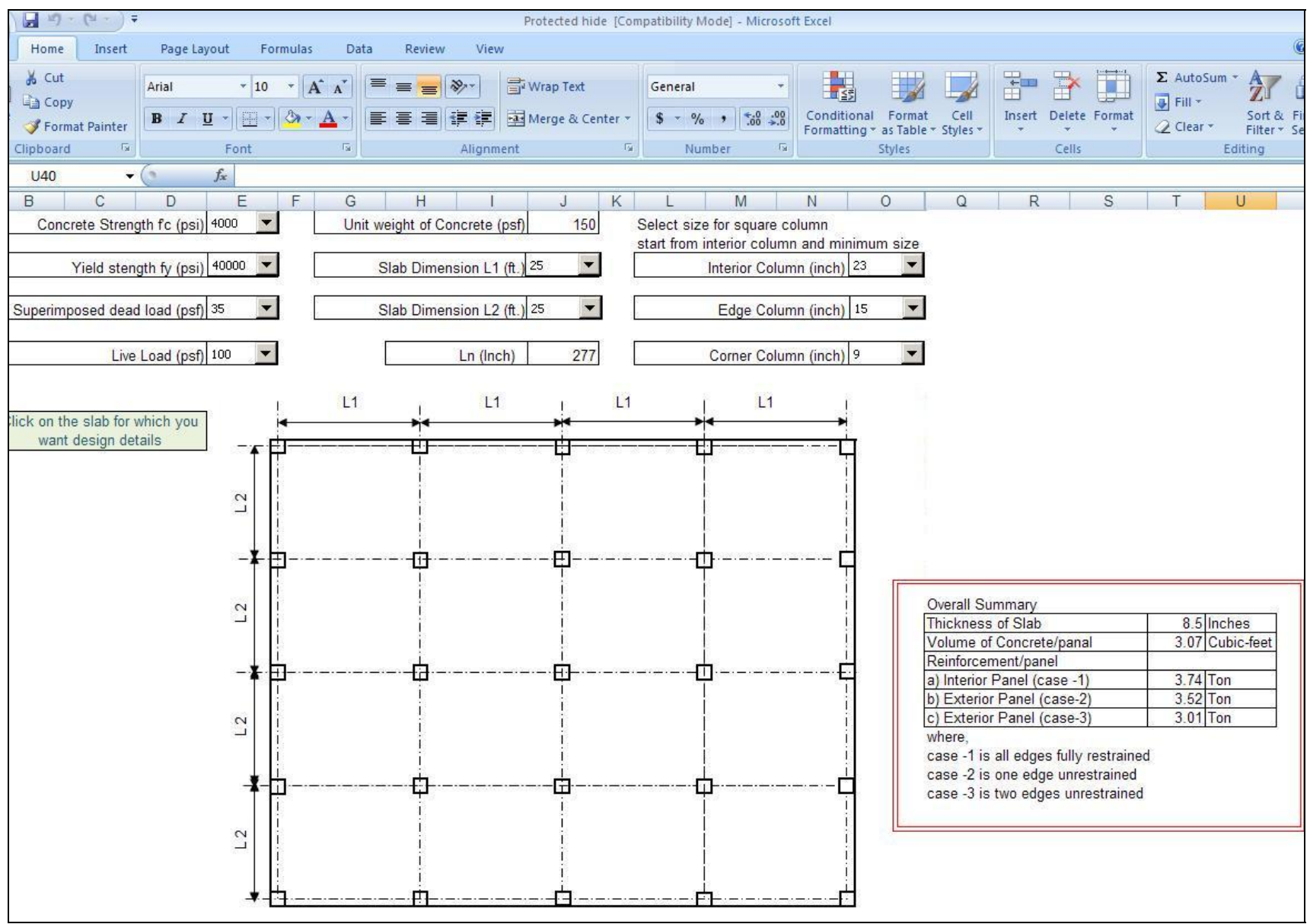

Figure 5 On Input sheet, summary of required steel and concrete

At the top of sheet we can see several drops down boxes, for selection of all design parameters which include geometrical and physical properties of flat slab elements. At bottom right corner we can see a table which summarizes the required tones of steel and volume of concrete per panel. Now user will have access to direct effects of changing parameter values on quantity of steel and concrete, which can be used to play with the values until optimal value is reached.

Basically user work is minimized to one sheet only. As per the given project description, one has to make just few changes in the default values of parameters and within a second, estimated values will be provided. This estimation is accurate as it takes values directly from actual design. This doesn't require any engineering knowledge, sales personal familiar with ms excel can also do the job real quickly. It saves all the design and estimation time at the preliminary stage of project. 


\section{Summary}

This paper describes a knowledge based excel system to automate the design and estimation of concrete flat slab. Input for basic design variables is taken from the user, by providing suitable guideline for selection. Design is checked to be technically safe for given material and geometrical properties. Detailed reinforcement analysis is done for 3 different panels of slab. User is provided with freedom to adjust sizes of bars according to number of bars and spacing. Due to the ability of excel to house the design method and code specifications a very quick design and estimation is done. Slab drawings and its respective reinforcement table can directly be used in making the bar cutting schedule and also in actual placement of bar. This approach can be used to fasten similar kind of preconstruction activities.

\section{References}

American Concrete Institute 318 - 08 Building Code Requirements for Structural Concrete and Commentary (2008). Portland Cement Association, Illinois

Best practice guides for in situ concrete frame buildings (2001). British Cement Association, Berkshire. Retrieved February 3, 2008 from http://projects.bre.co.uk/ConDiv/concrete\%20frame/Flat_Slabs_4pp_Web_pages.PDF

Karve, S. R., Shah, V. L. (2003). Limit state theory and design of reinforced concrete.

MacGregor, James G. \& Wight, James K. (2005) Prentice Hall Publication, New Jersey

Mehdi, S., \& Darvas, R., (2007). Concrete Structure. Prentice Hall Publication, New Jersey

Mousa, Tabatabai- Gargari, \& Elzarka, Hazem M. (1999) Integrated CAD/KBS Approach for Automating Preconstruction Activities. Journal of Construction Engineering and Management. 124(4), pp. 257-262.

VARDHAMAN V. BORA

Mr. Vardhaman V. Bora is a graduate student of the Department of Construction Science, Texas A\&M University.

MOHAMMED E. HAQUE, Ph.D., P.E.

Dr. Mohammed E. Haque is a professor in Construction Science, Texas A\&M University at College Station, Texas. He has over twenty years of professional experience in analysis, design, and investigation of building, bridges and tunnel structural projects of various city and state governments and private sectors. Dr. Haque is a registered Professional Engineer in the states of New York, Pennsylvania and Michigan, and members of ASEE, ASCE, and ACI. Dr. Haque received a BSCE from Bangladesh University of Engineering and Technology, a MSCE and a Ph.D. in Civil/Structural Engineering from New Jersey Institute of Technology, Newark, New Jersey. His research interests include fracture mechanics of engineering materials, composite materials and advanced construction materials, architectural/construction visualization and animation, computer applications in structural analysis and design, artificial neural network applications, knowledge based expert system developments, application based software developments, and buildings/ infrastructure/ bridges/tunnels inspection and database management systems. 